\title{
On some Hermite-Hadamard type inequalities for tgs-convex functions via generalized fractional integrals
}

\author{
Naila Mehreen ${ }^{1 *}$ (D) and Matloob Anwar'
}

\section{"Correspondence:}

nailamehreen@gmail.com

'School of Natural Sciences,

National University of Sciences and

Technology, Islamabad, Pakistan

\section{Springer}

\begin{abstract}
In this research article, we establish some Hermite-Hadamard type inequalities for tgs-convex functions via Katugampola fractional integrals and $\psi$-Riemann-Liouville fractional integrals. Through these results we give some new Hermite-Hadamard type inequalities for tgs-convex functions via Riemann-Liouville fractional integrals and classical integrals.
\end{abstract}

Keywords: Hermite-Hadamard inequality; tgs-convex functions; Riemann-Liouville fractional integrals; Katugampola fractional integrals; $\psi$-Riemann-Liouville fractional integrals

\section{Introduction}

The convex function and its generalization play an important role in optimization theory and in other field of sciences. These functions have many integral inequalities (see $[1,10$, 16]). The Hermite-Hadamard inequality $[4,5]$ for convex functions $\chi: \mathcal{H} \rightarrow \mathbb{R}$ on an interval $\mathcal{H}$ of the real line is defined by

$$
\chi\left(\frac{h_{1}+h_{2}}{2}\right) \leq \frac{1}{h_{2}-h_{1}} \int_{h_{1}}^{h_{2}} \chi(g) d g \leq \frac{\chi\left(h_{1}\right)+\chi\left(h_{2}\right)}{2},
$$

for all $h_{1}, h_{2} \in \mathcal{H}$ with $h_{1}<h_{2}$. Several applications are found by using the HermiteHadamard inequality (see $[2,3,6,12,14])$.

Fractional calculus [8] has played a key role in different scientific fields due to its long term memory methods. In [15], Sarikaya et al. proved some Hermite-Hadamard type integral inequalities for fractional integrals and also gave some applications. In $[10,11,13]$, the authors have established several Hermite-Hadamard type inequalities for new fractional conformable integral operators, Katugampola fractional integrals and $\psi$-RiemannLiouville fractional integrals, respectively.

Motivated by Liu et al. [9] and by [11,13], we prove Hermite-Hadamard type inequalities using $\psi$-Riemann-Liouville fractional integrals and Katugampola fractional integrals.

(c) The Author(s) 2020. This article is licensed under a Creative Commons Attribution 4.0 International License, which permits use, sharing, adaptation, distribution and reproduction in any medium or format, as long as you give appropriate credit to the original author(s) and the source, provide a link to the Creative Commons licence, and indicate if changes were made. The images or other third party material in this article are included in the article's Creative Commons licence, unless indicated otherwise in a credit line to the material. If material is not included in the article's Creative Commons licence and your intended use is not permitted by statutory regulation or exceeds the permitted use, you will need to obtain permission directly from the copyright holder. To view a copy of this licence, visit http://creativecommons.org/licenses/by/4.0/. 


\section{Preliminaries}

In this section, we give some definitions and relevant results essential for this research article.

Definition 2.1 ([18]) Let $\chi: \mathcal{H} \subseteq \mathbb{R} \rightarrow \mathbb{R}$ be a nonnegative function. Then $\chi$ is called tgs-convex, if it satisfies the following inequality:

$$
\chi\left(r h_{1}+(1-r) h_{2}\right) \leq r(1-r)\left[\chi\left(h_{1}\right)+\chi\left(h_{2}\right)\right]
$$

for all $h_{1}, h_{2} \in \mathcal{H}$ and $r \in[0,1]$.

Definition 2.2 ([8]) Let $\chi \in L\left[h_{1}, h_{2}\right]$. The right-hand side and left-hand side RiemannLiouville fractional integrals $J_{h_{1}+}^{\alpha} \chi$ and $J_{h_{2}-}^{\alpha} \chi$ of order $\alpha>0$ with $h_{2}>h_{1} \geq 0$ are defined by

$$
J_{h_{1}+}^{\alpha} \chi(g)=\frac{1}{\Gamma(\alpha)} \int_{h_{1}}^{g}(g-t)^{\alpha-1} \chi(t) d t, \quad g>h_{1}
$$

and

$$
J_{h_{2}-}^{\alpha} \chi(g)=\frac{1}{\Gamma(\alpha)} \int_{g}^{h_{2}}(t-g)^{\alpha-1} \chi(t) d t, \quad g<h_{2}
$$

respectively, where $\Gamma(\cdot)$ is the Gamma function defined by $\Gamma(\alpha)=\int_{0}^{\infty} e^{-t} t^{\alpha-1} d t$.

Definition 2.3 ([7]) Let $\left[h_{1}, h_{2}\right] \subset \mathbb{R}$ be a finite interval. Then, the left- and right-side Katugampola fractional integrals of order $\alpha(>0)$ of $\chi \in X_{c}^{p}\left(h_{1}, h_{2}\right)$ are defined by

$$
{ }^{\rho} I_{h_{1}+}^{\alpha} \chi(g)=\frac{\rho^{1-\alpha}}{\Gamma(\alpha)} \int_{h_{1}}^{g}\left(g^{\rho}-t^{\rho}\right)^{\alpha-1} t^{\rho-1} \chi(t) d t
$$

and

$$
{ }^{\rho} I_{h_{2}-}^{\alpha} \chi(g)=\frac{\rho^{1-\alpha}}{\Gamma(\alpha)} \int_{g}^{h_{2}}\left(t^{\rho}-g^{\rho}\right)^{\alpha-1} t^{\rho-1} \chi(t) d t
$$

with $h_{1}<g<h_{2}$ and $\rho>0$. Here $X_{c}^{p}\left(h_{1}, h_{2}\right)(c \in \mathbb{R}, 1 \leq p \leq \infty)$ is the space of those complex

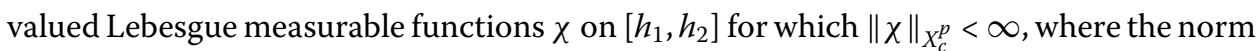
is defined by

$$
\|\chi\|_{X_{c}^{p}}=\left(\int_{h_{1}}^{h_{2}}\left|t^{c} \chi(t)\right|^{p} \frac{d t}{t}\right)^{1 / p}<\infty
$$

for $1 \leq p<\infty, c \in \mathbb{R}$ and, for the case $p=\infty$,

$$
\|\chi\|_{X_{c}^{\infty}}=\underset{h_{1} \leq t \leq h_{2}}{\operatorname{ess} \sup }\left[t^{c}|\chi(t)|\right] .
$$

Here ess sup stands for essential supremum. 
Definition 2.4 $([8,17])$ Let $\left(h_{1}, h_{2}\right)\left(-\infty \leq h_{1}<h_{2} \leq \infty\right)$ be a finite or infinite real interval and $\gamma>0$. Let $\psi(x)$ be an increasing and positive monotone function on $\left(h_{1}, h_{2}\right]$ with continuous derivative on $\left(h_{1}, h_{2}\right)$. Then the left- and right-sided $\psi$-Riemann-Liouville fractional integrals of a function $\chi$ with respect to $\psi$ on $\left[h_{1}, h_{2}\right]$ are defined by

$$
\begin{aligned}
& \mathcal{I}_{h_{1}+}^{\gamma: \psi} \chi(g)=\frac{1}{\Gamma(\gamma)} \int_{h_{1}}^{g} \psi^{\prime}(z)(\psi(g)-\psi(z))^{\gamma-1} \chi(z) d z, \\
& \mathcal{I}_{h_{2}-}^{\gamma: \psi} \chi(g)=\frac{1}{\Gamma(\gamma)} \int_{g}^{h_{2}} \psi^{\prime}(z)(\psi(z)-\psi(g))^{\gamma-1} \chi(z) d z,
\end{aligned}
$$

respectively.

Liu et al. [9] established Hermite-Hadamard type inequalities via $\psi$-Riemann-Liouville fractional integrals for convex functions.

Lemma 2.1 ([9]) Let $\chi:\left[h_{1}, h_{2}\right] \rightarrow \mathbb{R}$ be a differentiable mapping, for $0 \leq h_{1}<h_{2}$, and $\chi \in L_{1}\left[h_{1}, h_{2}\right]$. Let $\psi(g)$ be an increasing and positive monotone function on $\left(h_{1}, h_{2}\right]$, with continuous derivative $\psi^{\prime}(g)$ on $\left(h_{1}, h_{2}\right)$ and $\gamma \in(0,1)$. Then the following equality for fractional integral holds:

$$
\begin{aligned}
& \frac{\chi\left(h_{1}\right)+\chi\left(h_{2}\right)}{2}-\frac{\Gamma(\gamma+1)}{2\left(h_{2}-h_{1}\right)^{\gamma}}\left[\mathcal{I}_{\psi^{-1}\left(h_{1}\right)+}^{\gamma: \psi}(\chi \circ \psi)\left(\psi^{-1}\left(h_{2}\right)\right)\right. \\
& \left.\quad+\mathcal{I}_{\psi^{-1}\left(h_{2}\right)-}^{\gamma: \psi}(\chi \circ \psi)\left(\psi^{-1}\left(h_{1}\right)\right)\right] \\
& =\frac{1}{2\left(h_{2}-h_{1}\right)^{\gamma}} \int_{\psi^{-1}\left(h_{1}\right)}^{\psi^{-1}\left(h_{2}\right)}\left[\left(\psi(g)-h_{1}\right)^{\gamma}-\left(h_{2}-\psi(g)\right)^{\gamma}\right]\left(\chi^{\prime} \circ \psi\right)(g) \psi^{\prime}(g) d g .
\end{aligned}
$$

Lemma 2.2 ([9]) Let $\chi:\left[h_{1}, h_{2}\right] \rightarrow \mathbb{R}$ be a differentiable mapping, for $0 \leq h_{1}<h_{2}$, and $\chi \in L_{1}\left[h_{1}, h_{2}\right]$. Let $\psi(g)$ be an increasing and positive monotone function on $\left(h_{1}, h_{2}\right]$, with continuous derivative $\psi^{\prime}(g)$ on $\left(h_{1}, h_{2}\right)$ and $\gamma \in(0,1)$. Then the following equality for fractional integral holds:

$$
\begin{aligned}
& \frac{\Gamma(\gamma+1)}{2\left(h_{2}-h_{1}\right)^{\gamma}}\left[\mathcal{I}_{\psi^{-1}\left(h_{1}\right)+}^{\gamma: \psi}(\chi \circ \psi)\left(\psi^{-1}\left(h_{2}\right)\right)+\mathcal{I}_{\psi^{-1}\left(h_{2}\right)-}^{\gamma: \psi}(\chi \circ \psi)\left(\psi^{-1}\left(h_{1}\right)\right)\right] \\
& \quad-\chi\left(\frac{h_{1}+h_{2}}{2}\right) \\
& =\int_{\psi^{-1}\left(h_{1}\right)}^{\psi^{-1}\left(h_{2}\right)} k\left(\chi^{\prime} \circ \psi\right)(g) \psi^{\prime}(g) d g \\
& \quad+\frac{1}{2\left(h_{2}-h_{1}\right)^{\gamma}} \int_{\psi^{-1}\left(h_{1}\right)}^{\psi^{-1}\left(h_{2}\right)}\left[\left(\psi(g)-h_{1}\right)^{\gamma}-\left(h_{2}-\psi(g)\right)^{\gamma}\right]\left(\chi^{\prime} \circ \psi\right)(g) \psi^{\prime}(g) d g,
\end{aligned}
$$

where

$$
k= \begin{cases}\frac{1}{2}, & \psi^{-1}\left(\frac{h_{1}+h_{2}}{2}\right) \leq z \leq \psi^{-1}\left(h_{2}\right), \\ -\frac{1}{2}, & \psi^{-1}\left(h_{1}\right)<z<\psi^{-1}\left(\frac{h_{1}+h_{2}}{2}\right) .\end{cases}
$$




\section{Inequalities via Katugampola fractional integrals}

In this section, we find a Hermite-Hadamard inequality for a tgs-convex function via Katugampola fractional integrals.

Theorem 3.1 Let $\alpha>0$ and $\rho>0$. Let $\chi:\left[h_{1}^{\rho}, h_{2}^{\rho}\right] \subset \mathbb{R} \rightarrow \mathbb{R}$ be a nonnegative function with $0 \leq h_{1}<h_{2}$ and $\chi \in X_{c}^{p}\left(h_{1}^{\rho}, h_{2}^{\rho}\right)$. If $\chi$ is also a tgs-convex function on $\left[h_{1}^{\rho}, h_{2}^{\rho}\right]$, then the following inequalities hold:

$$
\begin{aligned}
& 2 \chi\left(\frac{h_{1}^{\rho}+h_{2}^{\rho}}{2}\right) \\
& \quad \leq \frac{\rho^{\alpha} \Gamma(\alpha+1)}{2\left(h_{2}^{\rho}-h_{1}^{\rho}\right)^{\alpha}}\left[{ }^{\rho} I_{h_{1}+}^{\alpha} \chi\left(h_{2}^{\rho}\right)+{ }^{\rho} I_{h_{2}-}^{\alpha} \chi\left(h_{1}^{\rho}\right)\right] \\
& \quad \leq \frac{\alpha\left(\chi\left(h_{1}^{\rho}\right)+\chi\left(h_{2}^{\rho}\right)\right)}{\rho(\alpha+1)(\alpha+2)} .
\end{aligned}
$$

Proof Let $r \in[0,1]$. Consider $x, y \in\left[h_{1}, h_{2}\right], h_{1} \geq 0$, defined by $x^{\rho}=r^{\rho} h_{1}^{\rho}+\left(1-r^{\rho}\right) h_{2}^{\rho}, y^{\rho}=$ $r^{\rho} h_{2}^{\rho}+\left(1-r^{\rho}\right) h_{1}^{\rho}$. Since $\chi$ is a tgs-convex function on $\left[h_{1}^{\rho}, h_{2}^{\rho}\right]$, we have

$$
\chi\left(\frac{x^{\rho}+y^{\rho}}{2}\right) \leq \frac{\chi\left(x^{\rho}\right)+\chi\left(y^{\rho}\right)}{4} .
$$

Then we have

$$
4 \chi\left(\frac{h_{1}^{\rho}+h_{2}^{\rho}}{2}\right) \leq \chi\left(r^{\rho} h_{1}^{\rho}+\left(1-r^{\rho}\right) h_{2}^{\rho}\right)+\chi\left(r^{\rho} h_{2}^{\rho}+\left(1-r^{\rho}\right) h_{1}^{\rho}\right) .
$$

Multiplying both sides of (6) by $r^{\alpha \rho-1}, \alpha>0$ and then integrating the resulting inequality with respect to $r$ over $[0,1]$, we obtain

$$
\begin{aligned}
\frac{4}{\alpha \rho} \chi\left(\frac{h_{1}^{\rho}+h_{2}^{\rho}}{2}\right) \leq & \int_{0}^{1} r^{\alpha \rho-1} \chi\left(r^{\rho} h_{1}^{\rho}+\left(1-r^{\rho}\right) h_{2}^{\rho}\right) d r \\
& +\int_{0}^{1} r^{\alpha \rho-1} \chi\left(r^{\rho} h_{2}^{\rho}+\left(1-r^{\rho}\right) h_{1}^{\rho}\right) d r \\
= & \int_{h_{2}}^{h_{1}}\left(\frac{h_{2}^{\rho}-g^{\rho}}{h_{2}^{\rho}-h_{1}^{\rho}}\right)^{\alpha-1} \chi\left(g^{\rho}\right) \frac{g^{\rho-1}}{h_{1}^{\rho}-h_{2}^{\rho}} d g \\
& +\int_{h_{1}}^{h_{2}}\left(\frac{k^{\rho}-h_{1}^{\rho}}{h_{2}^{\rho}-h_{1}^{\rho}}\right)^{\alpha-1} \chi\left(k^{\rho}\right) \frac{k^{\rho-1}}{h_{2}^{\rho}-h_{1}^{\rho}} d k \\
= & \frac{\rho^{\alpha-1} \Gamma(\alpha)}{\left(h_{2}^{\rho}-h_{1}^{\rho}\right)^{\alpha}}\left[{ }^{\rho} I_{h_{1}}^{\alpha} \chi\left(h_{2}^{\rho}\right)+{ }^{\rho} I_{h_{2}}^{\alpha} \chi\left(h_{1}^{\rho}\right)\right] .
\end{aligned}
$$

This establishes the first inequality. For the proof of the second inequality in (5), we first observe that, for a tgs-convex function $\chi$, we have

$$
\chi\left(r^{\rho} h_{1}^{\rho}+\left(1-r^{\rho}\right) h_{2}^{\rho}\right) \leq r^{\rho}\left(1-r^{\rho}\right)\left(\chi\left(h_{1}^{\rho}\right)+\chi\left(h_{2}^{\rho}\right)\right)
$$

and

$$
\chi\left(r^{\rho} h_{2}^{\rho}+\left(1-r^{\rho}\right) h_{1}^{\rho}\right) \leq r^{\rho}\left(1-r^{\rho}\right)\left(\chi\left(h_{1}^{\rho}\right)+\chi\left(h_{2}^{\rho}\right)\right) .
$$


By adding these inequalities, we get

$$
\chi\left(r^{\rho} h_{1}^{\rho}+\left(1-r^{\rho}\right) h_{2}^{\rho}\right)+\chi\left(r^{\rho} h_{2}^{\rho}+\left(1-r^{\rho}\right) h_{1}^{\rho}\right) \leq 2 r^{\rho}\left(1-r^{\rho}\right)\left(\chi\left(h_{1}^{\rho}\right)+\chi\left(h_{2}^{\rho}\right)\right) .
$$

Multiplying both sides of (8) by $r^{\alpha \rho-1}, \alpha>0$ and then integrating the resulting inequality with respect to $r$ over $[0,1]$, we obtain

$$
\frac{\rho^{\alpha-1} \Gamma(\alpha)}{\left(h_{2}^{\rho}-h_{1}^{\rho}\right)^{\alpha}}\left[{ }^{\rho} I_{h_{1}+}^{\alpha} \chi\left(h_{2}^{\rho}\right)+{ }^{\rho} I_{h_{2}-}^{\alpha} \chi\left(h_{1}^{\rho}\right)\right] \leq 2 \int_{0}^{1} r^{\alpha \rho+\rho-1}\left(1-r^{\rho}\right)\left(\chi\left(h_{1}^{\rho}\right)+\chi\left(h_{2}^{\rho}\right)\right) d r .
$$

Since

$$
\int_{0}^{1}\left(r^{\alpha \rho+\rho-1}-r^{\alpha \rho+2 \rho-1}\right) d t=\frac{1}{\rho(\alpha+1)(\alpha+2)}
$$

(9) becomes

$$
\frac{\rho^{\alpha-1} \Gamma(\alpha)}{\left(h_{2}^{\rho}-h_{1}^{\rho}\right)^{\alpha}}\left[{ }^{\rho} I_{h_{1}+}^{\alpha} \chi\left(h_{2}^{\rho}\right)+{ }^{\rho} I_{h_{2}-}^{\alpha} \chi\left(h_{1}^{\rho}\right)\right] \leq \frac{2\left(\chi\left(h_{1}^{\rho}\right)+\chi\left(h_{2}^{\rho}\right)\right)}{\rho(\alpha+1)(\alpha+2)} .
$$

Thus (7) and (10) give (5).

Remark 3.1 (1) By letting $\rho \rightarrow 1$ in (5) of Theorem 3.1 we get inequality 3.1 of Theorem 3.1 in [18].

(2) By letting $\rho \rightarrow 1$ and $\alpha=1$ in (5) of Theorem 3.1 we get inequality 2.2 of Theorem 2.1 in [18].

Theorem 3.2 Let $\alpha>0$ and $\rho>0$. Let $\chi:\left[h_{1}^{\rho}, h_{2}^{\rho}\right] \subset \mathbb{R} \rightarrow \mathbb{R}$ be a differentiable and nonnegative mapping on $\left(h_{1}^{\rho}, h_{2}^{\rho}\right)$ with $0 \leq h_{1}<h_{2}$. If $\left|\chi^{\prime}\right|$ is tgs-convex on $\left[h_{1}^{\rho}, h_{2}^{\rho}\right]$, then the following inequality holds:

$$
\begin{aligned}
& \left|\frac{\chi\left(h_{1}^{\rho}\right)+\chi\left(h_{2}^{\rho}\right)}{2}-\frac{\rho^{\alpha} \Gamma(\alpha+1)}{2\left(h_{2}^{\rho}-h_{1}^{\rho}\right)^{\alpha}}\left[{ }^{\rho} I_{h_{1}+}^{\alpha} \chi\left(h_{2}^{\rho}\right)+{ }^{\rho} I_{h_{2}}^{\alpha} \chi\left(h_{1}^{\rho}\right)\right]\right| \\
& \quad \leq \frac{h_{2}^{\rho}-h_{1}^{\rho}}{(\alpha+2)(\alpha+3)}\left[\left|\chi^{\prime}\left(h_{1}^{\rho}\right)\right|+\left|\chi^{\prime}\left(h_{2}^{\rho}\right)\right|\right] .
\end{aligned}
$$

Proof From (7) one can have

$$
\begin{aligned}
& \frac{\rho^{\alpha-1} \Gamma(\alpha)}{\left(h_{2}^{\rho}-h_{1}^{\rho}\right)^{\alpha}}\left[{ }^{\rho} I_{h_{1}+}^{\alpha} \chi\left(h_{2}^{\rho}\right)+{ }^{\rho} I_{h_{2}-}^{\alpha} \chi\left(h_{1}^{\rho}\right)\right] \\
& \quad=\int_{0}^{1} r^{\alpha \rho-1} \chi\left(r^{\rho} h_{1}^{\rho}+\left(1-r^{\rho}\right) h_{2}^{\rho}\right) d r+\int_{0}^{1} r^{\alpha \rho-1} \chi\left(r^{\rho} h_{2}^{\rho}+\left(1-r^{\rho}\right) h_{1}^{\rho}\right) d r .
\end{aligned}
$$

By integrating by parts, we then get

$$
\begin{aligned}
& \frac{\chi\left(h_{1}^{\rho}\right)+\chi\left(h_{2}^{\rho}\right)}{\alpha \rho}-\frac{\rho^{\alpha-1} \Gamma(\alpha)}{\left(h_{2}^{\rho}-h_{1}^{\rho}\right)^{\alpha}}\left[{ }^{\rho} I_{h_{1}}^{\alpha} \chi\left(h_{2}^{\rho}\right)+{ }^{\rho} I_{h_{2}-}^{\alpha} \chi\left(h_{1}^{\rho}\right)\right] \\
& \quad \frac{h_{2}^{\rho}-h_{1}^{\rho}}{\alpha} \int_{0}^{1} r^{\rho(\alpha+1)-1}\left[\chi^{\prime}\left(r^{\rho} h_{2}^{\rho}+\left(1-r^{\rho}\right) h_{1}^{\rho}\right)-\chi^{\prime}\left(r^{\rho} h_{1}^{\rho}+\left(1-r^{\rho}\right) h_{2}^{\rho}\right)\right] d r .
\end{aligned}
$$


By using the triangle inequality and the tgs-convexity of $\left|\chi^{\prime}\right|$, we obtain

$$
\begin{aligned}
& \left|\frac{\chi\left(h_{1}^{\rho}\right)+\chi\left(h_{2}^{\rho}\right)}{\alpha \rho}-\frac{\rho^{\alpha-1} \Gamma(\alpha)}{\left(h_{2}^{\rho}-h_{1}^{\rho}\right)^{\alpha}}\left[{ }^{\rho} I_{h_{1}+}^{\alpha} \chi\left(h_{2}^{\rho}\right)+{ }^{\rho} I_{h_{2}-}^{\alpha} \chi\left(h_{1}^{\rho}\right)\right]\right| \\
& \quad \leq \frac{h_{2}^{\rho}-h_{1}^{\rho}}{\alpha} \int_{0}^{1} r^{\rho(\alpha+1)-1}\left|\chi^{\prime}\left(r^{\rho} h_{2}^{\rho}+\left(1-r^{\rho}\right) h_{1}^{\rho}\right)-\chi^{\prime}\left(r^{\rho} h_{1}^{\rho}+\left(1-r^{\rho}\right) h_{2}^{\rho}\right)\right| d r \\
& \quad \leq \frac{h_{2}^{\rho}-h_{1}^{\rho}}{\alpha} \int_{0}^{1} r^{\rho(\alpha+1)-1}\left[\chi^{\prime}\left(r^{\rho} h_{2}^{\rho}+\left(1-r^{\rho}\right) h_{1}^{\rho}\right)+\chi^{\prime}\left(r^{\rho} h_{1}^{\rho}+\left(1-r^{\rho}\right) h_{2}^{\rho}\right)\right] d r \\
& \quad=\frac{2\left(h_{2}^{\rho}-h_{1}^{\rho}\right)}{\alpha} \int_{0}^{1} r^{\rho(\alpha+1)-1} r^{\rho}\left(1-r^{\rho}\right)\left[\left|\chi^{\prime}\left(h_{1}^{\rho}\right)\right|+\left|\chi^{\prime}\left(h_{2}^{\rho}\right)\right|\right] d r \\
& \quad=\frac{2\left(h_{2}^{\rho}-h_{1}^{\rho}\right)}{\alpha} \frac{\left|\chi^{\prime}\left(h_{1}^{\rho}\right)\right|+\left|\chi^{\prime}\left(h_{2}^{\rho}\right)\right|}{\rho(\alpha+2)(\alpha+3)} .
\end{aligned}
$$

Multiplying both sides of the above inequality by $\frac{\alpha \rho}{2}$, we get the required inequality (11).

Corollary 3.3 Consider the similar assumptions of Theorem 3.2.

1. If $\rho=1$, then

$$
\begin{aligned}
& \left|\frac{\chi\left(h_{1}\right)+\chi\left(h_{2}\right)}{2}-\frac{\Gamma(\alpha+1)}{2\left(h_{2}-h_{1}\right)^{\alpha}}\left[J_{h_{1}+}^{\alpha} \chi\left(h_{2}\right)+J_{h_{2}-}^{\alpha} \chi\left(h_{1}\right)\right]\right| \\
& \quad \leq \frac{h_{2}-h_{1}}{(\alpha+2)(\alpha+3)}\left[\left|\chi^{\prime}\left(h_{1}\right)\right|+\left|\chi^{\prime}\left(h_{2}\right)\right|\right] .
\end{aligned}
$$

2. If $\rho=\alpha=1$, then

$$
\left|\frac{\chi\left(h_{1}\right)+\chi\left(h_{2}\right)}{2}-\frac{1}{h_{2}-h_{1}} \int_{h_{1}}^{h_{2}} \chi(g) d g\right| \leq \frac{h_{2}-h_{1}}{12}\left[\left|\chi^{\prime}\left(h_{1}\right)\right|+\left|\chi^{\prime}\left(h_{2}\right)\right|\right] .
$$

For more results we need the following lemma, also proved in [11].

Lemma 3.1 ([11]) Let $\alpha>0$ and $\rho>0$. Let $\chi:\left[h_{1}^{\rho}, h_{2}^{\rho}\right] \subset \mathbb{R}_{+}=[0, \infty) \rightarrow \mathbb{R}$ be a differentiable mapping on $\left(h_{1}^{\rho}, h_{2}^{\rho}\right)$ with $0 \leq h_{1}<h_{2}$. Then the following equality holds if the fractional integrals exist:

$$
\begin{aligned}
& \frac{\chi\left(h_{1}^{\rho}\right)+\chi\left(h_{2}^{\rho}\right)}{2}-\frac{\rho^{\alpha} \Gamma(\alpha+1)}{2\left(h_{2}^{\rho}-h_{1}^{\rho}\right)^{\alpha}}\left[{ }^{\rho} I_{h_{1}+}^{\alpha} \chi\left(h_{2}^{\rho}\right)+{ }^{\rho} I_{h_{2}-}^{\alpha} \chi\left(h_{1}^{\rho}\right)\right] \\
& \quad=\frac{\rho\left(h_{2}^{\rho}-h_{1}^{\rho}\right)}{2} \int_{0}^{1}\left[\left(1-r^{\rho}\right)^{\alpha}-\left(r^{\rho}\right)^{\alpha}\right] r^{\rho-1} \chi^{\prime}\left(r^{\rho} h_{1}^{\rho}+\left(1-r^{\rho}\right) h_{2}^{\rho}\right) d r
\end{aligned}
$$

Proof By using the similar arguments as in the proof of Lemma 2 in [15]. First consider

$$
\begin{aligned}
\int_{0}^{1}\left(1-r^{\rho}\right)^{\alpha} r^{\rho-1} \chi^{\prime}\left(r^{\rho} h_{1}^{\rho}+\left(1-r^{\rho}\right) h_{2}^{\rho}\right) d r \\
=\left.\frac{\left(1-r^{\rho}\right)^{\alpha} \chi\left(r^{\rho} h_{1}^{\rho}+\left(1-r^{\rho}\right) h_{2}^{\rho}\right)}{\rho\left(h_{1}^{\rho}-h_{2}^{\rho}\right)}\right|_{0} ^{1} \\
\quad+\frac{\alpha}{h_{1}^{\rho}-h_{2}^{\rho}} \int_{0}^{1}\left(1-r^{\rho}\right)^{\alpha-1} r^{\rho-1} \chi\left(r^{\rho} h_{1}^{\rho}+\left(1-r^{\rho}\right) h_{2}^{\rho}\right) d r
\end{aligned}
$$




$$
\begin{aligned}
& =\frac{\chi\left(h_{2}^{\rho}\right)}{\rho\left(h_{2}^{\rho}-h_{1}^{\rho}\right)}-\frac{\alpha}{h_{2}^{\rho}-h_{1}^{\rho}} \int_{h_{2}}^{h_{1}}\left(\frac{g^{\rho}-h_{1}^{\rho}}{h_{2}^{\rho}-h_{1}^{\rho}}\right)^{\alpha-1} \cdot \frac{g^{\rho-1}}{h_{1}^{\rho}-h_{2}^{\rho}} d g \\
& =\frac{\chi\left(h_{2}^{\rho}\right)}{\rho\left(h_{2}^{\rho}-h_{1}^{\rho}\right)}-\left.\frac{\rho^{\alpha-1} \Gamma(\alpha+1)}{\left(h_{2}^{\rho}-h_{1}^{\rho}\right)^{\alpha+1}} \cdot{ }^{\rho} I_{h_{2}-}^{\alpha} \chi\left(g^{\rho}\right)\right|_{g=h_{1}} .
\end{aligned}
$$

Similarly, we can show that

$$
\begin{aligned}
& \int_{0}^{1} r^{\rho \alpha} \cdot r^{\rho-1} \chi^{\prime}\left(r^{\rho} h_{1}^{\rho}+\left(1-r^{\rho}\right) h_{2}^{\rho}\right) d r \\
& \quad=-\frac{\chi\left(h_{1}^{\rho}\right)}{\rho\left(h_{2}^{\rho}-h_{1}^{\rho}\right)}+\left.\frac{\rho^{\alpha-1} \Gamma(\alpha+1)}{\left(h_{2}^{\rho}-h_{1}^{\rho}\right)^{\alpha+1}} \cdot{ }^{\rho} I_{h_{1}+}^{\alpha} \chi\left(g^{\rho}\right)\right|_{g=h_{2}} .
\end{aligned}
$$

Thus from (18) and (19) we get (17).

Theorem 3.4 Let $\alpha>0$ and $\rho>0$. Let $\chi:\left[h_{1}^{\rho}, h_{2}^{\rho}\right] \subset \mathbb{R}_{+} \rightarrow \mathbb{R}$ be a differentiable and nonnegative mapping on $\left(h_{1}^{\rho}, h_{2}^{\rho}\right)$ such that $\chi^{\prime} \in L_{1}\left[h_{1}, h_{2}\right]$ with $0 \leq h_{1}<h_{2}$. If $\left|\chi^{\prime}\right|^{q}$ is tgs-convex on $\left[h_{1}^{\rho}, h_{2}^{\rho}\right]$ for some fixed $q \geq 1$, then the following inequality holds:

$$
\begin{aligned}
& \left|\frac{\chi\left(h_{1}^{\rho}\right)+\chi\left(h_{2}^{\rho}\right)}{2}-\frac{\rho^{\alpha} \Gamma(\alpha+1)}{2\left(h_{2}^{\rho}-h_{1}^{\rho}\right)^{\alpha}}\left[{ }^{\rho} I_{h_{1}+}^{\alpha} \chi\left(h_{2}^{\rho}\right)+{ }^{\rho} I_{h_{2}-}^{\alpha} \chi\left(h_{1}^{\rho}\right)\right]\right| \\
& \leq \frac{\left(h_{2}^{\rho}-h_{1}^{\rho}\right)}{2}\left(\frac{2}{\alpha+1}\right)^{1-1 / q} \\
& \quad \times\left(\left[\beta(2, \alpha+2)+\frac{1}{(\alpha+2)(\alpha+3)}\right]\left[\left|\chi^{\prime}\left(h_{1}^{\rho}\right)\right|^{q}+\left|\chi^{\prime}\left(h_{2}^{\rho}\right)\right|^{q}\right]\right)^{1 / q} .
\end{aligned}
$$

Proof Using Lemma 3.1 and the power mean inequality and the tgs-convexity of $\left|\chi^{\prime}\right|^{q}$, we obtain

$$
\begin{aligned}
\left|I_{\chi}\left(\alpha, \rho, h_{1}, h_{2}\right)\right| & \left|\frac{\rho\left(h_{2}^{\rho}-h_{1}^{\rho}\right)}{2} \int_{0}^{1}\left\{\left(1-r^{\rho}\right)^{\alpha}-\left(r^{\rho}\right)^{\alpha}\right\} r^{\rho-1} \chi^{\prime}\left(r^{\rho} h_{1}^{\rho}+\left(1-r^{\rho}\right) h_{2}^{\rho}\right) d r\right| \\
\leq & \frac{\rho\left(h_{2}^{\rho}-h_{1}^{\rho}\right)}{2}\left(\int_{0}^{1}\left|\left(1-r^{\rho}\right)^{\alpha}-\left(r^{\rho}\right)^{\alpha}\right| r^{\rho-1} d r\right)^{1-1 / q} \\
& \times\left(\int_{0}^{1}\left|\left(1-r^{\rho}\right)^{\alpha}-\left(r^{\rho}\right)^{\alpha}\right| r^{\rho-1}\left|\chi^{\prime}\left(r^{\rho} h_{1}^{\rho}+\left(1-r^{\rho}\right) h_{2}^{\rho}\right)\right|^{q} d r\right)^{1 / q} \\
\leq & \frac{\rho\left(h_{2}^{\rho}-h_{1}^{\rho}\right)}{2}\left(\int_{0}^{1}\left\{\left(1-r^{\rho}\right)^{\alpha}+\left(r^{\rho}\right)^{\alpha}\right\} r^{\rho-1} d r\right)^{1-1 / q} \\
& \times\left(\int_{0}^{1}\left\{\left(1-r^{\rho}\right)^{\alpha}+\left(r^{\rho}\right)^{\alpha}\right\} r^{\rho-1} r^{\rho}\left(1-r^{\rho}\right)\left[\left|\chi^{\prime}\left(h_{1}^{\rho}\right)\right|^{q}+\left|\chi^{\prime}\left(h_{2}^{\rho}\right)\right|^{q}\right] d r\right)^{1 / q} .
\end{aligned}
$$

By using the change of variable $t=r^{\rho}$, we get

$$
\begin{aligned}
& \int_{0}^{1}\left\{\left(1-r^{\rho}\right)^{\alpha}+\left(r^{\rho}\right)^{\alpha}\right\} r^{\rho-1} d r \\
& \quad=\int_{0}^{1}\left(1-r^{\rho}\right)^{\alpha} r^{\rho-1} d r+\int_{0}^{1}\left(r^{\rho}\right)^{\alpha} r^{\rho-1} d r
\end{aligned}
$$




$$
\begin{aligned}
& \quad=\frac{2}{\rho(\alpha+1)}, \\
& \int_{0}^{1}\left\{\left(1-r^{\rho}\right)^{\alpha}+\left(r^{\rho}\right)^{\alpha}\right\} r^{\rho-1} r^{\rho}\left(1-r^{\rho}\right) d r \\
& \quad=\int_{0}^{1}\left(1-r^{\rho}\right)^{\alpha} r^{\rho-1} r^{\rho}\left(1-r^{\rho}\right) d r+\int_{0}^{1}\left(r^{\rho}\right)^{\alpha} r^{\rho-1} r^{\rho}\left(1-r^{\rho}\right) d r \\
& =\frac{1}{\rho} \beta(2, \alpha+2)+\frac{1}{\rho(\alpha+2)(\alpha+3)} .
\end{aligned}
$$

Hence using (23) and (22) in (21) we get (20).

Corollary 3.5 Consider the similar assumptions of Theorem 3.4.

1. If $\rho=1$, then

$$
\begin{aligned}
& \left|\frac{\chi\left(h_{1}\right)+\chi\left(h_{2}\right)}{2}-\frac{\Gamma(\alpha+1)}{2\left(h_{2}-h_{1}\right)^{\alpha}}\left[J_{h_{1}+}^{\alpha} \chi\left(h_{2}\right)+J_{h_{2}-}^{\alpha} \chi\left(h_{1}\right)\right]\right| \\
& \leq \frac{\left(h_{2}-h_{1}\right)}{2}\left(\frac{2}{\alpha+1}\right)^{1-1 / q} \\
& \quad \times\left(\left[\beta(2, \alpha+2)+\frac{1}{(\alpha+2)(\alpha+3)}\right]\left[\left|\chi^{\prime}\left(h_{1}\right)\right|^{q}+\left|\chi^{\prime}\left(h_{2}\right)\right|^{q}\right]\right)^{1 / q} .
\end{aligned}
$$

2. If $\rho=\alpha=1$, then

$$
\begin{aligned}
& \left|\frac{\chi\left(h_{1}\right)+\chi\left(h_{2}\right)}{2}-\frac{1}{h_{2}-h_{1}} \int_{h_{1}}^{h_{2}} \chi(g) d g\right| \\
& \quad \leq \frac{\left(h_{2}-h_{1}\right)}{2}\left(\frac{2\left(\left|\chi^{\prime}\left(h_{1}\right)\right|^{q}+\left|\chi^{\prime}\left(h_{2}\right)\right|^{q}\right)}{3}\right)^{1 / q} .
\end{aligned}
$$

Theorem 3.6 Let $\alpha>0$ and $\rho>0$. Let $\chi:\left[h_{1}^{\rho}, h_{2}^{\rho}\right] \subset \mathbb{R}_{+} \rightarrow \mathbb{R}$ be a differentiable and nonnegative mapping on $\left(h_{1}^{\rho}, h_{2}^{\rho}\right)$ such that $\chi^{\prime} \in L_{1}\left[h_{1}, h_{2}\right]$ with $0 \leq h_{1}<h_{2}$. If $\left|\chi^{\prime}\right|^{q}$ is tgs-convex on $\left[h_{1}^{\rho}, h_{2}^{\rho}\right]$ for some fixed $q \geq 1$, then the following inequality holds:

$$
\begin{aligned}
& \left|\frac{\chi\left(h_{1}^{\rho}\right)+\chi\left(h_{2}^{\rho}\right)}{2}-\frac{\rho^{\alpha} \Gamma(\alpha+1)}{2\left(h_{2}^{\rho}-h_{1}^{\rho}\right)^{\alpha}}\left[{ }^{\rho} I_{h_{1}+}^{\alpha} \chi\left(h_{2}^{\rho}\right)+{ }^{\rho} I_{h_{2}-}^{\alpha} \chi\left(h_{1}^{\rho}\right)\right]\right| \\
& \quad \leq \frac{\left(h_{2}^{\rho}-h_{1}^{\rho}\right)}{2}\left(\left[\beta(2, \alpha+2)+\frac{1}{(\alpha+2)(\alpha+3)}\right]\left[\left|\chi^{\prime}\left(h_{1}^{\rho}\right)\right|^{q}+\left|\chi^{\prime}\left(h_{2}^{\rho}\right)\right|^{q}\right]\right)^{1 / q} .
\end{aligned}
$$

Proof Using Lemma 3.1 and the power mean inequality and the tgs-convexity of $\left|\chi^{\prime}\right|^{q}$, we obtain

$$
\begin{aligned}
& \left|\frac{\chi\left(h_{1}^{\rho}\right)+\chi\left(h_{2}^{\rho}\right)}{2}-\frac{\rho^{\alpha} \Gamma(\alpha+1)}{2\left(h_{2}^{\rho}-h_{1}^{\rho}\right)^{\alpha}}\left[{ }^{\rho} I_{h_{1}+}^{\alpha} \chi\left(h_{2}^{\rho}\right)+{ }^{\rho} I_{h_{2}-}^{\alpha} \chi\left(h_{1}^{\rho}\right)\right]\right| \\
& \quad=\left|\frac{\rho\left(h_{2}^{\rho}-h_{1}^{\rho}\right)}{2} \int_{0}^{1}\left\{\left(1-r^{\rho}\right)^{\alpha}-\left(r^{\rho}\right)^{\alpha}\right\} r^{\rho-1} \chi^{\prime}\left(r^{\rho} h_{1}^{\rho}+\left(1-r^{\rho}\right) h_{2}^{\rho}\right) d r\right| \\
& \quad \leq \frac{\rho\left(h_{2}^{\rho}-h_{1}^{\rho}\right)}{2}\left(\int_{0}^{1} r^{\rho-1} d r\right)^{1-1 / q}
\end{aligned}
$$




$$
\begin{aligned}
& \times\left(\int_{0}^{1}\left|\left(1-r^{\rho}\right)^{\alpha}-\left(r^{\rho}\right)^{\alpha}\right| r^{\rho-1}\left|\chi^{\prime}\left(r^{\rho} h_{1}^{\rho}+\left(1-r^{\rho}\right) h_{2}^{\rho}\right)\right|^{q} d r\right)^{1 / q} \\
\leq & \frac{\rho\left(h_{2}^{\rho}-h_{1}^{\rho}\right)}{2}\left(\frac{1}{\rho}\right)^{1-1 / q} \\
& \times\left(\int_{0}^{1}\left\{\left(1-r^{\rho}\right)^{\alpha}+\left(r^{\rho}\right)^{\alpha}\right\} r^{\rho-1} r^{\rho}\left(1-r^{\rho}\right)\left[\left|\chi^{\prime}\left(h_{1}^{\rho}\right)\right|^{q}+\left|\chi^{\prime}\left(h_{2}^{\rho}\right)\right|^{q}\right] d r\right)^{1 / q} .
\end{aligned}
$$

Since by using the change of variable $t=r^{\rho}$, we get

$$
\begin{aligned}
& \int_{0}^{1}\left\{\left(1-r^{\rho}\right)^{\alpha}+\left(r^{\rho}\right)^{\alpha}\right\} r^{\rho-1} r^{\rho}\left(1-r^{\rho}\right) d r \\
& \quad=\int_{0}^{1}\left(1-r^{\rho}\right)^{\alpha} r^{\rho-1} r^{\rho}\left(1-r^{\rho}\right) d r+\int_{0}^{1}\left(r^{\rho}\right)^{\alpha} r^{\rho-1} r^{\rho}\left(1-r^{\rho}\right) d r \\
& \quad=\frac{1}{\rho} \beta(2, \alpha+2)+\frac{1}{\rho(\alpha+2)(\alpha+3)} .
\end{aligned}
$$

Hence using (28) in (27) we get(26).

Corollary 3.7 Consider the similar assumptions of Theorem 3.6. If $\rho=1$, then

$$
\begin{aligned}
& \left|\frac{\chi\left(h_{1}\right)+\chi\left(h_{2}\right)}{2}-\frac{\Gamma(\alpha+1)}{2\left(h_{2}-h_{1}\right)^{\alpha}}\left[J_{h_{1}+}^{\alpha} \chi\left(h_{2}\right)+J_{h_{2}-}^{\alpha} \chi\left(h_{1}\right)\right]\right| \\
& \quad \leq \frac{\left(h_{2}-h_{1}\right)}{2}\left(\left[\beta(2, \alpha+2)+\frac{1}{(\alpha+2)(\alpha+3)}\right]\left[\left|\chi^{\prime}\left(h_{1}\right)\right|^{q}+\left|\chi^{\prime}\left(h_{2}\right)\right|^{q}\right]\right)^{1 / q} .
\end{aligned}
$$

Theorem 3.8 Let $\chi_{1}, \chi_{2}$ be real valued, symmetric about $\frac{h_{1}^{\rho}+h_{2}^{\rho}}{2}$, nonnegative and tgsconvex functions on $\left[h_{1}^{\rho}, h_{2}^{\rho}\right]$, where $\rho>0$. Then, for all $h_{1}, h_{2}>0$ and $\alpha>0$, we have

$$
\frac{\rho^{\alpha \rho} I_{h_{1}+}^{\alpha}\left(\chi_{1}\left(h_{2}^{\rho}\right) \chi_{2}\left(h_{2}^{\rho}\right)\right)}{\left(h_{2}^{\rho}-h_{1}^{\rho}\right)^{\alpha}} \leq \frac{2 \alpha(\alpha+1)\left[M\left(h_{1}^{\rho}, h_{2}^{\rho}\right)+N\left(h_{1}^{\rho}, h_{2}^{\rho}\right)\right]}{\Gamma(\alpha+5)}
$$

and

$$
\begin{aligned}
8 & \chi_{1}\left(\frac{h_{1}^{\rho}+h_{2}^{\rho}}{2}\right) \chi_{2}\left(\frac{h_{1}^{\rho}+h_{2}^{\rho}}{2}\right) \\
& \leq \frac{\rho^{\alpha} \rho I_{h_{1}+}^{\alpha}\left(\chi_{1}\left(h_{2}^{\rho}\right) \chi_{2}\left(h_{2}^{\rho}\right)\right)}{\left(h_{2}^{\rho}-h_{1}^{\rho}\right)^{\alpha}}+\frac{2 \alpha(\alpha+1)\left[M\left(h_{1}^{\rho}, h_{2}^{\rho}\right)+N\left(h_{1}^{\rho}, h_{2}^{\rho}\right)\right]}{\Gamma(\alpha+5)},
\end{aligned}
$$

where $M\left(h_{1}^{\rho}, h_{2}^{\rho}\right)=\chi_{1}\left(h_{1}\right) \chi_{2}\left(h_{1}\right)+\chi_{1}\left(h_{2}\right) \chi_{2}\left(h_{2}\right)$ and $N\left(h_{1}^{\rho}, h_{2}^{\rho}\right)=\chi_{1}\left(h_{1}\right) \chi_{2}\left(h_{2}\right)+\chi_{1}\left(h_{2}\right) \chi_{2}\left(h_{1}\right)$.

Proof Since $\chi_{1}$ and $\chi_{2}$ are tgs-convex functions on $\left[h_{1}, h_{2}\right]$, we can have

$$
\chi_{1}\left(r^{\rho} h_{1}^{\rho}+\left(1-r^{\rho}\right) h_{2}^{\rho}\right) \leq r^{\rho}\left(1-r^{\rho}\right)\left(\chi_{1}\left(h_{1}^{\rho}\right)+\chi_{1}\left(h_{2}^{\rho}\right)\right)
$$

and

$$
\chi_{2}\left(r^{\rho} h_{1}^{\rho}+\left(1-r^{\rho}\right) h_{2}^{\rho}\right) \leq r^{\rho}\left(1-r^{\rho}\right)\left(\chi_{2}\left(h_{1}^{\rho}\right)+\chi_{2}\left(h_{2}^{\rho}\right)\right),
$$


From the above, we obtain

$$
\begin{aligned}
& \chi_{1}\left(r^{\rho} h_{1}^{\rho}+\left(1-r^{\rho}\right) h_{2}^{\rho}\right) \chi_{2}\left(r^{\rho} h_{1}^{\rho}+\left(1-r^{\rho}\right) h_{2}^{\rho}\right) \\
& \quad \leq r^{2 \rho}\left(1-r^{\rho}\right)^{2}\left(\chi_{1}\left(h_{1}^{\rho}\right)+\chi_{1}\left(h_{2}^{\rho}\right)\right)\left(\chi_{2}\left(h_{1}^{\rho}\right)+\chi_{2}\left(h_{2}^{\rho}\right)\right) .
\end{aligned}
$$

Multiplying both sides of (32) by $\frac{r^{\alpha \rho-1}}{\Gamma(\alpha)}, \alpha>0$ and then integrating the resulting inequality with respect to $r$ over $[0,1]$, we obtain

$$
\begin{gathered}
\frac{1}{\Gamma(\alpha)} \int_{0}^{1} r^{\alpha \rho-1} \chi_{1}\left(r^{\rho} h_{1}^{\rho}+\left(1-r^{\rho}\right) h_{2}^{\rho}\right) \chi_{2}\left(r^{\rho} h_{1}^{\rho}+\left(1-r^{\rho}\right) h_{2}^{\rho}\right) d r \\
\quad \leq \frac{\left(\chi_{1}\left(h_{1}^{\rho}\right)+\chi_{1}\left(h_{2}^{\rho}\right)\right)\left(\chi_{2}\left(h_{1}^{\rho}\right)+\chi_{2}\left(h_{2}^{\rho}\right)\right)}{\Gamma(\alpha)} \int_{0}^{1} r^{2 \rho}\left(1-r^{\rho}\right)^{2} d r .
\end{gathered}
$$

By the change of variable $t=r^{\rho}$, we get

$$
\int_{0}^{1} r^{2 \rho}\left(1-r^{\rho}\right)^{2} d r=\frac{2 \alpha(\alpha+1)}{\rho \Gamma(\alpha+5)} .
$$

Also by letting $x^{\rho}=r^{\rho} h_{1}^{\rho}+\left(1-r^{\rho}\right) h_{2}^{\rho}$, we obtain

$$
\begin{aligned}
& \frac{1}{\Gamma(\alpha)} \int_{0}^{1} r^{\alpha \rho-1} \chi_{1}\left(r^{\rho} h_{1}^{\rho}+\left(1-r^{\rho}\right) h_{2}^{\rho}\right) \chi_{2}\left(r^{\rho} h_{1}^{\rho}+\left(1-r^{\rho}\right) h_{2}^{\rho}\right) d r \\
& \quad=\frac{\rho^{\alpha-1}{ }^{\rho} I_{h_{1}+}^{\alpha}\left(\chi_{1}\left(h_{2}^{\rho}\right) \chi_{2}\left(h_{2}^{\rho}\right)\right)}{\left(h_{2}^{\rho}-h_{1}^{\rho}\right)^{\alpha}} .
\end{aligned}
$$

Hence from (33)-(35), we get (30).

Again using the tgs-convexity of $\chi_{1}$ and $\chi_{2}$ on $\left[h_{1}^{\rho}, h_{2}^{\rho}\right]$, we find

$$
\begin{aligned}
\chi_{1}( & \left.\frac{h_{1}^{\rho}+h_{2}^{\rho}}{2}\right) \chi_{2}\left(\frac{h_{1}^{\rho}+h_{2}^{\rho}}{2}\right) \\
\leq & \chi_{1}\left(\frac{r^{\rho} h_{1}^{\rho}+\left(1-r^{\rho}\right) h_{2}^{\rho}}{2}+\frac{r^{\rho} h_{2}^{\rho}+\left(1-r^{\rho}\right) h_{1}^{\rho}}{2}\right) \\
& \times \chi_{2}\left(\frac{r^{\rho} h_{1}^{\rho}+\left(1-r^{\rho}\right) h_{2}^{\rho}}{2}+\frac{r^{\rho} h_{2}^{\rho}+\left(1-r^{\rho}\right) h_{1}^{\rho}}{2}\right) \\
\leq & \frac{1}{4}\left[\chi_{1}\left(r^{\rho} h_{1}^{\rho}+\left(1-r^{\rho}\right) h_{2}^{\rho}\right)+\chi_{1}\left(r^{\rho} h_{2}^{\rho}+\left(1-r^{\rho}\right) h_{1}^{\rho}\right)\right] \\
& \times \frac{1}{4}\left[\chi_{2}\left(r^{\rho} h_{1}^{\rho}+\left(1-r^{\rho}\right) h_{2}^{\rho}\right)+\chi_{1}\left(r^{\rho} h_{2}^{\rho}+\left(1-r^{\rho}\right) h_{1}^{\rho}\right)\right] \\
= & \frac{1}{16}\left[\chi _ { 1 } ( r ^ { \rho } h _ { 1 } ^ { \rho } + ( 1 - r ^ { \rho } ) h _ { 2 } ^ { \rho } ) \chi _ { 2 } \left(r^{\rho} h_{1}^{\rho}+\left(1-r^{\rho}\right) h_{2}^{\rho}\right.\right. \\
& +\chi_{1}\left(r^{\rho} h_{2}^{\rho}+\left(1-r^{\rho}\right) h_{1}^{\rho}\right) \chi_{2}\left(r^{\rho} h_{2}^{\rho}+\left(1-r^{\rho}\right) h_{1}^{\rho}\right. \\
& +\chi_{1}\left(r^{\rho} h_{1}^{\rho}+\left(1-r^{\rho}\right) h_{2}^{\rho}\right) \chi_{2}\left(r^{\rho} h_{2}^{\rho}+\left(1-r^{\rho}\right) h_{1}^{\rho}\right. \\
& \left.+\chi_{1}\left(r^{\rho} h_{2}^{\rho}+\left(1-r^{\rho}\right) h_{1}^{\rho}\right) \chi_{2}\left(r^{\rho} h_{1}^{\rho}+\left(1-r^{\rho}\right) h_{2}^{\rho}\right)\right] .
\end{aligned}
$$


Multiplying both sides of (36) by $\frac{r^{\alpha \rho-1}}{\Gamma(\alpha)}, \alpha>0$ and then integrating the resulting inequality with respect to $r$ over $[0,1]$, we obtain

$$
\begin{aligned}
& \frac{1}{\rho \Gamma(\alpha+1)} \chi_{1}\left(\frac{h_{1}^{\rho}+h_{2}^{\rho}}{2}\right) \chi_{2}\left(\frac{h_{1}^{\rho}+h_{2}^{\rho}}{2}\right) \\
& \leq \frac{1}{16 \Gamma(\alpha)}\left[\int _ { 0 } ^ { 1 } r ^ { \alpha \rho - 1 } \chi _ { 1 } ( r ^ { \rho } h _ { 1 } ^ { \rho } + ( 1 - r ^ { \rho } ) h _ { 2 } ^ { \rho } ) \chi _ { 2 } \left(r^{\rho} h_{1}^{\rho}+\left(1-r^{\rho}\right) h_{2}^{\rho} d r\right.\right. \\
& \quad+\int_{0}^{1} r^{\alpha \rho-1} \chi_{1}\left(r^{\rho} h_{2}^{\rho}+\left(1-r^{\rho}\right) h_{1}^{\rho}\right) \chi_{2}\left(r^{\rho} h_{2}^{\rho}+\left(1-r^{\rho}\right) h_{1}^{\rho} d r\right. \\
& \quad+\int_{0}^{1} r^{\alpha \rho-1} \chi_{1}\left(r^{\rho} h_{1}^{\rho}+\left(1-r^{\rho}\right) h_{2}^{\rho}\right) \chi_{2}\left(r^{\rho} h_{2}^{\rho}+\left(1-r^{\rho}\right) h_{1}^{\rho} d r\right. \\
& \left.\quad+\int_{0}^{1} r^{\alpha \rho-1} \chi_{1}\left(r^{\rho} h_{2}^{\rho}+\left(1-r^{\rho}\right) h_{1}^{\rho}\right) \chi_{2}\left(r^{\rho} h_{1}^{\rho}+\left(1-r^{\rho}\right) h_{2}^{\rho}\right) d r\right] .
\end{aligned}
$$

That is,

$$
\begin{aligned}
8 \chi_{1}\left(\frac{h_{1}^{\rho}+h_{2}^{\rho}}{2}\right) \chi_{2}\left(\frac{h_{1}^{\rho}+h_{2}^{\rho}}{2}\right) \\
\leq \frac{\rho \Gamma(\alpha+1)}{2\left(h_{2}^{\rho}-h_{1}^{\rho}\right)^{\alpha}}{ }^{\rho} I_{h_{1}+}^{\alpha}\left[\chi_{1}\left(h_{2}^{\rho}\right) \chi_{2}\left(h_{2}^{\rho}\right)+\chi_{1}\left(h_{2}^{\rho}\right) \chi_{2}\left(h_{1}^{\rho}\right)\right] \\
\quad+{ }^{\rho} I_{h_{1}+}^{\alpha}\left[\chi_{1}\left(h_{1}^{\rho}\right) \chi_{2}\left(h_{1}^{\rho}\right)+\chi_{1}\left(h_{1}^{\rho}\right) \chi_{2}\left(h_{2}^{\rho}\right)\right] .
\end{aligned}
$$

After some calculations we get the required inequality (31).

Remark 3.2 1. By letting $\rho=1$ in Theorem 3.8 the inequalities (30) and (31) give the inequalities (3.11) and (3.12), respectively, in Theorem 3.2 of [18].

2. By letting $\rho=\alpha=1$ in Theorem 3.8 the inequality (30) becomes the inequality in Theorem (2.2) of [18].

\section{Inequalities via $\psi$-Riemann-Liouville fractional integrals}

First we establish the Hermite-Hadamard inequality via $\psi$-Riemann-Liouville fractional integrals.

Theorem 4.1 Let $\chi:\left[h_{1}, h_{2}\right] \rightarrow \mathbb{R}$ be a positive function, for $0 \leq h_{1}<h_{2}$, and $\chi \in$ $L_{1}\left[h_{1}, h_{2}\right]$. Let $\psi(z)$ be an increasing and positive monotone function on $\left(h_{1}, h_{2}\right]$, with continuous derivative $\psi^{\prime}(z)$ on $\left(h_{1}, h_{2}\right)$. Let $\chi$ be a tgs-convex function, then the following inequalities for a fractional integral hold:

$$
\begin{aligned}
& 2 \chi\left(\frac{h_{1}+h_{2}}{2}\right) \\
& \quad \leq \frac{\Gamma(\gamma+1)}{2\left(h_{2}-h_{1}\right)^{\gamma}}\left[\mathcal{I}_{\psi^{-1}\left(h_{1}\right)+}^{\gamma: \psi}(\chi \circ \psi)\left(\psi^{-1}\left(h_{2}\right)\right)+\mathcal{I}_{\psi^{-1}\left(h_{2}\right)-}^{\gamma: \psi}(\chi \circ \psi)\left(\psi^{-1}\left(h_{1}\right)\right)\right] \\
& \quad \leq \frac{\gamma\left[\chi\left(h_{1}\right)+\chi\left(h_{2}\right)\right]}{(\gamma+1)(\gamma+2)} .
\end{aligned}
$$


Proof Since $\chi$ is tgs-convex, we have

$$
\chi\left(\frac{u+v}{2}\right) \leq \frac{\chi(u)+\chi(v)}{2^{2}}
$$

Let $u=r h_{1}+(1-r) h_{2}$ and $v=r h_{2}+(1-r) h_{1}$, we get

$$
4 \chi\left(\frac{h_{1}+h_{2}}{2}\right) \leq \chi\left(r h_{1}+(1-r) h_{2}\right)+\chi\left(r h_{2}+(1-r) h_{1}\right)
$$

Multiplying by $r^{\gamma-1}$ on both sides of inequality (38) and then integrating with respect to $r$ over $[0,1]$ imply

$$
\frac{4}{\gamma} \chi\left(\frac{h_{1}+h_{2}}{2}\right) \leq \int_{0}^{1} r^{\gamma-1} \chi\left(r h_{1}+(1-r) h_{2}\right) d r+\int_{0}^{1} r^{\gamma-1} \chi\left(r h_{2}+(1-r) h_{1}\right) d r .
$$

Now consider

$$
\begin{aligned}
\frac{\Gamma(\gamma+1)}{2\left(h_{2}-h_{1}\right)^{\gamma}}\left[\mathcal{I}_{\psi^{-1}\left(h_{1}\right)+}^{\gamma: \psi}(\chi \circ \psi)\left(\psi^{-1}\left(h_{2}\right)\right)+\mathcal{I}_{\psi^{-1}\left(h_{2}\right)-}^{\gamma: \psi}(\chi \circ \psi)\left(\psi^{-1}\left(h_{1}\right)\right)\right] \\
=\frac{\Gamma(\gamma+1)}{2\left(h_{2}-h_{1}\right)^{\gamma} \Gamma(\gamma)}\left[\int_{\psi^{-1}\left(h_{1}\right)}^{\psi^{-1}\left(h_{2}\right)} \psi^{\prime}(g)\left(h_{2}-\psi(g)\right)^{\gamma-1}(\chi \circ \psi)(g) d g\right. \\
\left.\quad+\int_{\psi^{-1}\left(h_{1}\right)}^{\psi^{-1}\left(h_{2}\right)} \psi^{\prime}(g)\left(\psi(g)-h_{1}\right)^{\gamma-1}(\chi \circ \psi)(g) d g\right] \\
=\frac{\gamma}{2}\left[\int_{\psi^{-1}\left(h_{1}\right)}^{\psi^{-1}\left(h_{2}\right)}\left(\frac{h_{2}-\psi(g)}{h_{2}-h_{1}}\right)^{\gamma-1} \chi(\psi(g)) \frac{\psi^{\prime}(g)}{h_{2}-h_{1}} d g\right. \\
\left.\quad+\int_{\psi^{-1}\left(h_{1}\right)}^{\psi^{-1}\left(h_{2}\right)}\left(\frac{\psi(g)-h_{1}}{h_{2}-h_{1}}\right)^{\gamma-1} \chi(\psi(g)) \frac{\psi^{\prime}(g)}{h_{2}-h_{1}} d g\right] \\
=\frac{\gamma}{2}\left[\int_{0}^{1} r^{\gamma-1} \chi\left(r h_{1}+(1-r) h_{2}\right) d r+\int_{0}^{1} r^{\gamma-1} \chi\left(r h_{2}+(1-r) h_{1}\right) d r\right] \\
\geq 2 \chi\left(\frac{h_{1}+h_{2}}{2}\right),
\end{aligned}
$$

by using (39). Thus first inequality of (37) is proved.

For the next inequality we consider

$$
\chi\left(r h_{1}+(1-r) h_{2}\right) \leq r(1-r)\left[\chi\left(h_{1}\right)+\chi\left(h_{2}\right)\right]
$$

and

$$
\chi\left(r h_{2}+(1-r) h_{1}\right) \leq r(1-r)\left[\chi\left(h_{2}\right)+\chi\left(h_{1}\right)\right]
$$

We add

$$
\chi\left(r h_{1}+(1-r) h_{2}\right)+\chi\left(r h_{2}+(1-r) h_{1}\right) \leq 2 r(1-r)\left[\chi\left(h_{1}\right)+\chi\left(h_{2}\right)\right] .
$$


Multiplying by $r^{\gamma-1}$ on both sides of inequality (41) and then integrating with respect to $r$ over $[0,1]$ imply

$$
\begin{aligned}
& \int_{0}^{1} r^{\gamma-1} \chi\left(r h_{1}+(1-r) h_{2}\right) d r+\int_{0}^{1} r^{\gamma-1} \chi\left(r h_{2}+(1-r) h_{1}\right) d r \\
& \quad \leq \frac{2\left[\chi\left(h_{1}\right)+\chi\left(h_{2}\right)\right]}{(\gamma+1)(\gamma+2)} .
\end{aligned}
$$

That is,

$$
\begin{aligned}
& \frac{\Gamma(\gamma+1)}{\left(h_{2}-h_{1}\right)^{\gamma}}\left[\mathcal{I}_{\psi^{-1}\left(h_{1}\right)+}^{\gamma: \psi}(\chi \circ \psi)\left(\psi^{-1}\left(h_{2}\right)\right)+\mathcal{I}_{\psi^{-1}\left(h_{2}\right)-}^{\gamma: \psi}(\chi \circ \psi)\left(\psi^{-1}\left(h_{1}\right)\right)\right] \\
& \quad \leq \frac{\gamma\left[\chi\left(h_{1}\right)+\chi\left(h_{2}\right)\right]}{(\gamma+1)(\gamma+2)} .
\end{aligned}
$$

Hence the proof is completed.

Remark 4.1 (1) By letting $\psi(g)=g$ in (37) of Theorem 4.1 we get inequality 3.1 of Theorem 3.1 in [18].

(2) By letting $\psi(g)=g$ and $\gamma=1$ in (37) of Theorem 4.1 we get inequality 2.2 of Theorem 2.1 in [18].

For the next two results we use Lemma 2.1 and Lemma 2.2, respectively.

Theorem 4.2 Let $\chi:\left[h_{1}, h_{2}\right] \rightarrow \mathbb{R}$ be a nonnegative differentiable mapping, for $0 \leq h_{1}<$ $h_{2}$. Let $\psi(g)$ be an increasing and positive monotone function on $\left(h_{1}, h_{2}\right]$, with continuous derivative $\psi^{\prime}(g)$ on $\left(h_{1}, h_{2}\right)$ and $\gamma \in(0,1)$. If $\left|\chi^{\prime}\right|^{q}$ is tgs-convex and $q \geq 1$, then the following inequality for fractional integral holds:

$$
\begin{aligned}
& \mid \frac{\chi\left(h_{1}\right)+\chi\left(h_{2}\right)}{2}-\frac{\Gamma(\gamma+1)}{2\left(h_{2}-h_{1}\right)^{\gamma}}\left[\mathcal{I}_{\psi^{-1}\left(h_{1}\right)+}^{\gamma: \psi}(\chi \circ \psi)\left(\psi^{-1}\left(h_{2}\right)\right)\right. \\
& \left.\quad+\mathcal{I}_{\psi^{-1}\left(h_{2}\right)-}^{\gamma: \psi}(\chi \circ \psi)\left(\psi^{-1}\left(h_{1}\right)\right)\right] \mid \\
& \leq \frac{h_{2}-h_{1}}{2}\left[\frac{2}{\gamma+1}\left(1-\frac{1}{2^{\gamma}}\right)\right]^{\frac{q-1}{q}}\left(\frac{2\left(\left|\chi^{\prime}\left(h_{1}\right)\right|^{q}+\left|\chi\left(h_{2}\right)\right|^{q}\right)}{(\gamma+2)(\gamma+3)}\right)^{\frac{1}{q}} .
\end{aligned}
$$

Proof First note that, for every $g \in\left(\psi^{-1}\left(h_{1}\right), \psi^{-1}\left(h_{2}\right)\right)$, we have $h_{1}<\psi(g)<h_{2}$. Let $r=$ $\frac{h_{2}-\psi(g)}{h_{2}-h_{1}}$, then we have $\psi(g)=r h_{1}+(1-r) h_{2}$. Applying Lemma 2.1 and the tgs-convexity of $\left|\chi^{\prime}\right|$, we obtain

$$
\begin{aligned}
& \mid \frac{\chi\left(h_{1}\right)+\chi\left(h_{2}\right)}{2}-\frac{\Gamma(\gamma+1)}{2\left(h_{2}-h_{1}\right)^{\gamma}}\left[\mathcal{I}_{\psi^{-1}\left(h_{1}\right)_{+}}^{\gamma: \psi}(\chi \circ \psi)\left(\psi^{-1}\left(h_{2}\right)\right)\right. \\
& \left.\quad+\mathcal{I}_{\psi^{-1}\left(h_{2}\right)-}^{\gamma: \psi}(\chi \circ \psi)\left(\psi^{-1}\left(h_{1}\right)\right)\right] \mid \\
& \quad \leq \frac{1}{2\left(h_{2}-h_{1}\right)^{\gamma}} \int_{\psi^{-1}\left(h_{1}\right)}^{\psi^{-1}\left(h_{2}\right)}\left|\left(\psi(g)-h_{1}\right)^{\gamma}-\left(h_{2}-\psi(g)\right)^{\gamma}\right|\left|\left(\chi^{\prime} \circ \psi\right)(g)\right| d \psi(g) \\
& \quad=\frac{h_{2}-h_{1}}{2} \int_{0}^{1}\left|(1-r)^{\gamma}-r^{\gamma}\right|\left|\chi^{\prime}\left(r h_{1}+(1-r) h_{2}\right)\right| d r
\end{aligned}
$$




$$
\begin{aligned}
& \leq \frac{h_{2}-h_{1}}{2} \int_{0}^{1}\left|(1-r)^{\gamma}-r^{\gamma}\right| r(1-r)\left[\left|\chi^{\prime}\left(h_{2}\right)+\right| \chi^{\prime}\left(h_{2}\right) \mid\right] d r \\
& \leq \frac{h_{2}-h_{1}}{2} \int_{0}^{1}\left[(1-r)^{\gamma}+r^{\gamma}\right] r(1-r)\left[\left|\chi^{\prime}\left(h_{2}\right)+\right| \chi^{\prime}\left(h_{2}\right) \mid\right] d r \\
& =\frac{h_{2}-h_{1}}{(\gamma+2)(\gamma+3)}\left[\left|\chi^{\prime}\left(h_{2}\right)+\right| \chi^{\prime}\left(h_{2}\right) \mid\right] .
\end{aligned}
$$

Since

$$
\int_{0}^{1}\left[(1-r)^{\gamma}+r^{\gamma}\right] r(1-r) d r=\frac{2}{(\gamma+2)(\gamma+3)},
$$

we get the required inequality (42) for $q=1$.

Now consider the case when $q>1$. Again using Lemma 2.1, the power mean inequality and the $s$-convexity of $\left|\chi^{\prime}\right|^{q}$ on $\left[a_{1}, a_{2}\right]$, we get

$$
\begin{aligned}
\mid \frac{\chi\left(h_{1}\right)+\chi\left(h_{2}\right)}{2}-\frac{\Gamma(\gamma+1)}{2\left(h_{2}-h_{1}\right)^{\gamma}}\left[\mathcal{I}_{\psi^{-1}\left(h_{1}\right)+}^{\gamma: \psi}(\chi \circ \psi)\left(\psi^{-1}\left(h_{2}\right)\right)\right. \\
\left.\quad+\mathcal{I}_{\psi^{-1}\left(h_{2}\right)-}^{\gamma: \psi}(\chi \circ \psi)\left(\psi^{-1}\left(h_{1}\right)\right)\right] \mid \\
\leq \frac{1}{2\left(h_{2}-h_{1}\right)^{\gamma}} \int_{\psi^{-1}\left(h_{1}\right)}^{\psi^{-1}\left(h_{2}\right)}\left|\left(\psi(g)-h_{1}\right)^{\gamma}-\left(h_{2}-\psi(g)\right)^{\gamma}\right|\left|\left(\chi^{\prime} \circ \psi\right)(g)\right| d \psi(g) \\
=\frac{h_{2}-h_{1}}{2} \int_{0}^{1}\left|(1-r)^{\gamma}-r^{\gamma}\right|\left|\chi^{\prime}\left(r h_{1}+(1-r) h_{2}\right)\right| d r \\
=\frac{h_{2}-h_{1}}{2}\left(\int_{0}^{1}\left|(1-r)^{\gamma}-r^{\gamma}\right| d r\right)^{1-\frac{1}{q}} \\
\quad \times\left(\int_{0}^{1}\left|(1-r)^{\gamma}-r^{\gamma}\right|\left|\chi^{\prime}\left(r h_{1}+(1-r) h_{2}\right)\right|^{q} d r\right)^{\frac{1}{q}} \\
=\frac{h_{2}-h_{1}}{2}\left(\int_{0}^{1}\left|(1-r)^{\gamma}-r^{\gamma}\right| d r\right)^{\frac{q-1}{q}} \\
\quad \times\left(\int_{0}^{1}\left[(1-r)^{\gamma}+r^{\gamma}\right] r(1-r)\left[\left|\chi^{\prime}\left(h_{2}\right)\right|^{q}+\left.\chi^{\prime}\left(h_{2}\right)\right|^{q}\right] d r\right)^{\frac{1}{q}} \\
=\frac{h_{2}-h_{1}}{2}\left[\frac{2}{\gamma+1}\left(1-\frac{1}{2^{\gamma}}\right)\right]^{\frac{q-1}{q}}\left(\frac{2\left(\left|\chi^{\prime}\left(h_{1}\right)\right|^{q}+\left|\chi\left(h_{2}\right)\right|^{q}\right)}{(\gamma+2)(\gamma+3)}\right)^{\frac{1}{q}} .
\end{aligned}
$$

We have

$$
\begin{aligned}
\int_{0}^{1}\left|(1-r)^{\gamma}-r^{\gamma}\right| d r & =\int_{0}^{1 / 2}\left[(1-r)^{\gamma}-r^{\gamma}\right] d r+\int_{1 / 2}^{1}\left[r^{\gamma}-(1-r)^{\gamma}\right] d r \\
& =\frac{2}{\gamma+1}\left(1-\frac{1}{2^{\gamma}}\right) .
\end{aligned}
$$

This completes the proof.

Corollary 4.3 Under the similar conditions of Theorem 4.2. 
1. If $\psi(g)=g$, then we get

$$
\begin{aligned}
& \left|\frac{\chi\left(h_{1}\right)+\chi\left(h_{2}\right)}{2}-\frac{\Gamma(\gamma+1)}{2\left(h_{2}-h_{1}\right)^{\gamma}}\left[J_{h_{1}+}^{\gamma} \chi\left(h_{2}\right)+J_{h_{2}-}^{\gamma} \chi\left(h_{1}\right)\right]\right| \\
& \quad \leq \frac{h_{2}-h_{1}}{2}\left[\frac{2}{\gamma+1}\left(1-\frac{1}{2^{\gamma}}\right)\right]^{\frac{q-1}{q}}\left(\frac{2\left(\left|\chi^{\prime}\left(h_{1}\right)\right|^{q}+\left|\chi\left(h_{2}\right)\right|^{q}\right)}{(\gamma+2)(\gamma+3)}\right)^{\frac{1}{q}} .
\end{aligned}
$$

2. If $\psi(g)=g$ and $\gamma=1$, then we get

$$
\begin{aligned}
& \left|\frac{\chi\left(h_{1}\right)+\chi\left(h_{2}\right)}{2}-\frac{2}{\left(h_{2}-h_{1}\right)} \int_{h_{1}}^{h_{2}} \chi(g) d g\right| \\
& \quad \leq \frac{h_{2}-h_{1}}{2}\left[\frac{1}{2}\right]^{\frac{q-1}{q}}\left(\frac{\left|\chi^{\prime}\left(h_{1}\right)\right|^{q}+\left|\chi\left(h_{2}\right)\right|^{q}}{3}\right)^{\frac{1}{q}} .
\end{aligned}
$$

Theorem 4.4 Let $\chi:\left[h_{1}, h_{2}\right] \rightarrow \mathbb{R}$ be a nonnegative differentiable mapping, for $0 \leq h_{1}<$ $h_{2}$. Let $\psi(g)$ be an increasing and positive monotone function on $\left(h_{1}, h_{2}\right]$, with continuous derivative $\psi^{\prime}(g)$ on $\left(h_{1}, h_{2}\right)$ and $\gamma \in(0,1)$. If $\left|\chi^{\prime}\right|$ is tgs-convex, then the following inequality for fractional integral holds:

$$
\begin{aligned}
& \mid \frac{\Gamma(\gamma+1)}{2\left(h_{2}-h_{1}\right)^{\gamma}}\left[\mathcal{I}_{\psi^{-1}\left(h_{1}\right)+}^{\gamma: \psi}(\chi \circ \psi)\left(\psi^{-1}\left(h_{2}\right)\right)+\mathcal{I}_{\psi^{-1}\left(h_{2}\right)-}^{\gamma: \psi}(\chi \circ \psi)\left(\psi^{-1}\left(h_{1}\right)\right)\right] \\
& \quad-\chi\left(\frac{h_{1}+h_{2}}{2}\right) \mid \\
& \quad \leq \frac{\chi\left(h_{2}\right)-\chi\left(h_{1}\right)}{2}+\frac{h_{2}-h_{1}}{(\gamma+2)(\gamma+3)}\left(\left|\chi^{\prime}\left(h_{1}\right)\right|+\left|\chi\left(h_{2}\right)\right|\right) .
\end{aligned}
$$

Proof From Lemma 2.2 and the tgs-convexity of $\left|\chi^{\prime}\right|$, we have

$$
\begin{aligned}
& \mid \frac{\Gamma(\gamma+1)}{2\left(h_{2}-h_{1}\right)^{\gamma}}\left[\mathcal{I}_{\psi^{-1}\left(h_{1}\right)+}^{\gamma: \psi}(\chi \circ \psi)\left(\psi^{-1}\left(h_{2}\right)\right)+\mathcal{I}_{\psi^{-1}\left(h_{2}\right)-}^{\gamma: \psi}(\chi \circ \psi)\left(\psi^{-1}\left(h_{1}\right)\right)\right] \\
& \quad-\chi\left(\frac{h_{1}+h_{2}}{2}\right) \mid \\
&=\mid \int_{\psi^{-1}\left(h_{1}\right)}^{\psi^{-1}\left(h_{2}\right)} k\left(\chi^{\prime} \circ \psi\right)(g) \psi^{\prime}(g) d g \\
& \quad+\frac{1}{2\left(h_{2}-h_{1}\right)^{\gamma}} \int_{\psi^{-1}\left(h_{1}\right)}^{\psi^{-1}\left(h_{2}\right)}\left[\left(\psi(g)-h_{1}\right)^{\gamma}-\left(h_{2}-\psi(g)\right)^{\gamma}\right]\left(\chi^{\prime} \circ \psi\right)(g) \psi^{\prime}(g) d g \mid \\
& \leq\left|\int_{\psi^{-1}\left(h_{1}\right)}^{\psi^{-1}\left(h_{2}\right)} k\left(\chi^{\prime} \circ \psi\right)(g) \psi^{\prime}(g) d g\right| \\
& \quad+\left|\frac{1}{2\left(h_{2}-h_{1}\right)^{\gamma}} \int_{\psi^{-1}\left(h_{1}\right)}^{\psi^{-1}\left(h_{2}\right)}\left[\left(\psi(z)-h_{1}\right)^{\gamma}-\left(h_{2}-\psi(g)\right)^{\gamma}\right]\left(\chi^{\prime} \circ \psi\right)(g) \psi^{\prime}(g) d g\right| \\
&:= S_{1}+S_{2},
\end{aligned}
$$

where

$$
S_{1}:=\left|\int_{\psi^{-1}\left(h_{1}\right)}^{\psi^{-1}\left(h_{2}\right)} k\left(\chi^{\prime} \circ \psi\right)(g) \psi^{\prime}(g) d g\right|
$$




$$
S_{2}:=\left|\frac{1}{2\left(h_{2}-h_{1}\right)^{\gamma}} \int_{\psi^{-1}\left(h_{1}\right)}^{\psi^{-1}\left(h_{2}\right)}\left[\left(\psi(z)-h_{1}\right)^{\gamma}-\left(h_{2}-\psi(g)\right)^{\gamma}\right]\left(\chi^{\prime} \circ \psi\right)(g) \psi^{\prime}(g) d g\right| \text {, }
$$

and $k$ is defined as in Lemma 2.2. Note that

$$
S_{1}=\frac{\chi\left(h_{2}\right)-\chi\left(h_{1}\right)}{2}
$$

and from Theorem 4.2 for the case $q=1$, we have

$$
S_{2} \leq \frac{h_{2}-h_{1}}{(\gamma+2)(\gamma+3)}\left(\left(\left|\chi^{\prime}\left(h_{1}\right)\right|+\left|\chi\left(h_{2}\right)\right|\right)\right) \text {. }
$$

Hence by using (49) and (50) in (48), we get (47).

Corollary 4.5 Assume the similar conditions of Theorem 4.4.

1. If $\psi(g)=g$, then we get

$$
\begin{aligned}
& \left|\frac{\Gamma(\gamma+1)}{2\left(h_{2}-h_{1}\right)^{\gamma}}\left[J_{h_{1}+}^{\gamma} \chi\left(h_{2}\right)+J_{h_{2}-}^{\gamma} \chi\left(h_{1}\right)\right]-\chi\left(\frac{h_{1}+h_{2}}{2}\right)\right| \\
& \quad \leq \frac{\chi\left(h_{2}\right)-\chi\left(h_{1}\right)}{2}+\frac{h_{2}-h_{1}}{(\gamma+2)(\gamma+3)}\left(\left(\left|\chi^{\prime}\left(h_{1}\right)\right|+\left|\chi\left(h_{2}\right)\right|\right)\right) .
\end{aligned}
$$

2. If $\psi(g)=g$ and $\gamma=1$, then we get

$$
\begin{aligned}
& \left|\frac{2}{\left(h_{2}-h_{1}\right)} \int_{h_{1}}^{h_{2}} \chi(g) d g-\chi\left(\frac{h_{1}+h_{2}}{2}\right)\right| \\
& \quad \leq \frac{\chi\left(h_{2}\right)-\chi\left(h_{1}\right)}{2}+\frac{h_{2}-h_{1}}{6}\left(\left|\chi^{\prime}\left(h_{1}\right)\right|+\left|\chi\left(h_{2}\right)\right|\right) .
\end{aligned}
$$

\section{Conclusion}

In this paper, we proved in Theorem 3.1 the Hermite-Hadamard inequality for tgs-convex functions via Katugampola fractional integrals. From Theorems 3.2-3.6, we established a Hermite-Hadamard type inequality for tgs-convex functions via Katugampola fractional integrals. From Corollaries 3.3 and 3.5 we obtained a new Hermite-Hadamard type inequality for $t g s$-convex functions via Riemann-Liouville fractional and classical integrals. Also from Corollary 3.7 we obtained a new Hermite-Hadamard type inequality for tgsconvex functions via Riemann-Liouville fractional integrals.

On the other hand, from Theorem 4.1 we obtained the Hermite-Hadamard inequality for tgs-convex functions via $\psi$-Riemann-Liouville fractional integrals. From Theorems 4.2 and 4.4, we established a Hermite-Hadamard type inequality for tgs-convex functions via $\psi$-Riemann-Liouville fractional integrals. From Corollaries 4.3 and 4.5 we obtained a new Hermite-Hadamard type inequality for tgs-convex functions via Riemann-Liouville fractional and classical integrals. 
Competing interests

The authors declare that they have no competing interests.

\section{Authors' contributions}

The two authors contributed equally to this work. Both authors read and approved the final manuscript.

\section{Publisher's Note}

Springer Nature remains neutral with regard to jurisdictional claims in published maps and institutional affiliations.

Received: 11 September 2019 Accepted: 9 December 2019 Published online: 03 January 2020

\section{References}

1. Alzabut, J., Abdeljawad, T., Jarad, F., Sudsutad, W.: A Gronwall inequality via the generalized proportional fractional derivative with applications. J. Inequal. Appl. 2019, 101 (2019)

2. Avci, M., Kavurmaci, H., Ozdemir, M.E.: New inequalities of Hermite-Hadamard type via s-convex functions in the second sense with applications. Appl. Math. Comput. 217, 5171-5176 (2011)

3. Chen, F., Wu, S.: Several complementary inequalities to inequalities of Hermite-Hadamard type for s-convex functions. J. Nonlinear Sci. Appl. 9, 705-716 (2016)

4. Hadamard, J.: Étude sur les propriétés des fonctions entières et en particulier d'une fonction considérée par Riemann. J. Math. Pures Appl., 171-215 (1893)

5. Hermite, C.: Sur deux limites d'une intégrale dénie. Mathesis 3, 82 (1883)

6. Iscan, I.: Hermite-Hadamard type inequalities for p-convex functions. Int. J. Anal. Appl. 11, 137-145 (2016)

7. Katugampola, U.N.: New approach to generalized fractional derivatives. Bull. Math. Anal. Appl. 6(4), 1-15 (2014)

8. Kilbas, A.A., Srivastava, H.M., Trujillo, J.J.: Theory and Applications of Fractional Differential Equations. Elsevier Science B. V., Amsterdam (2006)

9. Liu, K., Wang, J., O'Regan, D.: On the Hermite-Hadamard type inequality for $\psi$-Riemann-Liouville fractional integrals via convex functions. J. Inequal. Appl. (2019)

10. Mehreen, N., Anwar, M.: Hermite-Hadamard and Hermite-Hadamard-Fejer type inequalities for p-convex functions via new fractional conformable integral operators. J. Math. Comput. Sci. 19, 230-240 (2018)

11. Mehreen, N., Anwar, M.: Integral inequalities for some convex functions via generalized fractional integrals. J. Inequal. Appl. 2018, 208 (2018)

12. Mehreen, N., Anwar, M.: Hermite-Hadamard type inequalities via exponentially p-convex functions and exponentially s-convex functions in second sense with applications. J. Inequal. Appl. 2019, 92 (2019)

13. Mehreen, N., Anwar, M.: Some inequalities via $\psi$-Riemann-Liouville fractional integrals. AIMS Math. 4(5), 1403-1415 (2019)

14. Niculescu, C.P., Persson, L.-E.: Convex Functions and Their Applications. A Contemporary Approach, 2nd edn. Springer, Berlin (2018)

15. Sarikaya, M.Z., Set, E., Yaldiz, H., Basak, N.: Hermite-Hadamard's inequalities for fractional integrals and related fractional inequalities. Math. Comput. Model. 57(9), 2403-2407 (2013)

16. Set, E.: New inequalities of Ostrowski type for mappings whose derivatives are s-convex in the second sense via fractional integrals. Comput. Math. Appl. 63, 1147-1154 (2012)

17. Sousa, V.C., Oliveira, E.C.: On the $\psi$-Hilfer fractional derivative. Commun. Nonlinear Sci. Numer. Simul. 60, 72-91 (2018)

18. Tunc, M., Gov, E., Şanal, U.: On tgs-convex function and their inequalities. Facta Univ., Ser. Math. Inform. 30, 679-691 (2015)

\section{Submit your manuscript to a SpringerOpen ${ }^{\circ}$ journal and benefit from:}

- Convenient online submission

- Rigorous peer review

- Open access: articles freely available online

- High visibility within the field

Retaining the copyright to your article

Submit your next manuscript at $>$ springeropen.com 